\title{
In Vivo Imaging Technology of Transplanted Stem Cells Using Quantum Dots for Regenerative Medicine
}

\author{
Hiroshi YUKaWA ${ }^{* 1, * 2 \dagger}$ and Yoshinobu BABA $* 1, * 2, * 3, * 4 \dagger$ \\ *1 Department of Biomolecular Engineering, Graduate School of Engineering, Nagoya University, Furo-cho, \\ Chikusa, Nagoya 464-8603, Japan \\ *2 ImPACT Research Center for Advanced Nanobiodevices, Nagoya University, Furo-cho, Chikusa, \\ Nagoya 464-8603, Japan \\ *3 Institute of Innovation for Future Society, Nagoya University, Furo-cho, Chikusa, Nagoya 464-8603, Japan \\ *4 Health Research Institute, National Institute of Advanced Industrial Science and Technology (AIST), \\ 2217-14 Hayashi-cho, Takamatsu 761-0395, Japan
}

\begin{abstract}
Quantum dots (QDs) have excellent fluorescence properties in comparison to traditional fluorescence probes. Thus, the optical application of QDs is rapidly expanding to each field of analytical chemistry. In this review paper, we reviewed the application of QDs to regenerative medicine, especially stem cell transplantation therapy. The labeling of stem cells using QDs composed of semiconductor materials in combination with a chemical substance, poly-cationic liposome and cell penetrating peptide is reported. In addition, the influence of QD labeling on the pluripotency of stem cells is also reported. Finally, the in vivo imaging of transplanted stem cells in mice by QDs emitting fluorescence in the near-infrared region, which can be detected by in vivo fluorescence imaging systems such as IVIS and SAI-1000, is described. The future prospects for stem cell imaging technology by QDs are also discussed.
\end{abstract}

Keywords Quantum dots (QDs), stem cells, induced pluripotent stem cells (iPS cells), in vivo imaging

(Received October 27, 2017; Accepted January 16, 2018; Published May 10, 2018)

\begin{tabular}{|c|c|c|c|}
\hline 1 Introduction & 526 & Labeled with QDs & 528 \\
\hline 2 QD Types and Stem Cell Labeling Strategies & & 5 In Vivo NIR-II Fluorescence Imaging Systems & \\
\hline Using QDs & 526 & and Probes & 530 \\
\hline 3 Influence of QD Labeling on the Pluripotency of & & 6 Conclusions & 531 \\
\hline Stem Cells & 527 & 7 Acknowledgements & 531 \\
\hline 4 In Vivo Fluorescence Imaging of Stem Cells & & 8 References & 531 \\
\hline
\end{tabular}

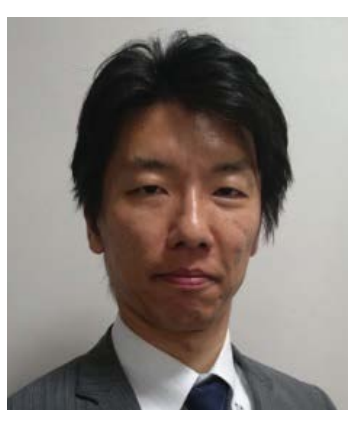

Hiroshi Yukawa is a Designated Associate Professor of the Department of Biomolecular Engineering, Graduate School of Engineering, Nagoya University. $\mathrm{He}$ obtained a Master degree in Engineering from Tokyo University, Japan in 2002. He joined Drug Development Group, R\&D Company, KOBAYASHI Pharmaceutical Co., Ltd. in 2002. He earned his Ph.D. in Medical Science from Nagoya University in 2010 under the supervision of Prof. M. Hamaguchi, and earned his Ph.D. in Engineering from Tokyo University in 2011 under the supervision of Prof. K. Araki. He has been employed in the Graduate School of Engineering at Nagoya University from 2012.

$\dagger$ To whom correspondence should be addressed. E-mail: h.yukawa@nanobio.nagoya-u.ac.jp (H. Y.); babaymtt@ chembio.nagoya-u.ac.jp (Y. B.)

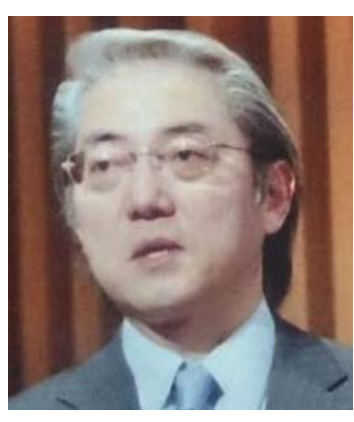

Yoshinobu BABA is a Professor of the Department of Biomolecular Engineering, Graduate School of Engineering, Nagoya University. $\mathrm{He}$ is an Associate Editor of Analytical Chemistry and serves as an editorial/advisory board member for over 15 scientific journals. He has received over 160 awards for his contribution in the field of nanotechnology. He is the author or co-author of 931 publications and is also an inventor of over 90 patents. $\mathrm{He}$ has delivered more than 875 plenaries and invited lectures at conferences. His work has been cited on 356 occasions by newspapers and television. 


\section{Introduction}

Regenerative medicine is drawing much attention for application to the treatment of diseases that are difficult to treat with conventional therapies such as organ transplantation and pharmacotherapy. Stem cell therapy in particular plays an important role in regenerative medicine for a number of diseases such as lung and liver diseases because of difficulties in regenerating those organs. ${ }^{1-4}$ Therefore, technology for detecting transplanted stem cells in vivo with high sensitivity is essential for ensuring the safety and therapeutic effect of stem cell therapy. ${ }^{5,6}$ However, in vivo imaging modalities capable of detecting transplanted stem cells remain to be established.

There are some major problems that prevent the successful in vivo fluorescence imaging of transplanted stem cells at high resolution. Those probes include the strong scattering, absorption and autofluorescence derived from the living organism. In order to overcome these problems, fluorescence probes that can absorb the excitation light and emit strong fluorescence in the near-infrared region (NIR) (NIR-I: about $700-900 \mathrm{~nm}$, NIR-II: about $1000-1600 \mathrm{~nm}$ ) and in the "Biological Optical Window" to decrease the scattering, absorption and autofluorescence derived from the body, are strongly desired.
Quantum dots (QDs) with smart fluorescence properties have received focus as a potential alternative to conventional fluorescence probes for use in stem cell imaging. ${ }^{7-9}$ QDs have already demonstrated super-high resolution, super-long duration, super-high sensitivity and energy savings, so they were applied to $4 \mathrm{~K}$ displays in 2013. ${ }^{10}$ Moreover, QDs showing strong fluorescence in the near-infrared region have been developed and are commercially available. QDs are thus expected to be useful for in vivo fluorescence imaging of transplanted stem cells. Stem cell labeling technologies using QDs and in vivo fluorescence imaging technologies of transplanted stem cells have been explored by our group since 2008.11-26 In this review paper, we mainly overview our research topics, including QD types, stem cell labeling methods by QDs and in vivo fluorescence imaging of transplanted stem cells labeled with QDs.

\section{QD Types and Stem Cell Labeling Strategies Using QDs}

QDs have been used as inorganic fluorescence probes in bioassays such as enzyme-linked immune sorbent assay (ELISA), because QDs have a number of advantages over protein or organic probes. Those advantages include high a

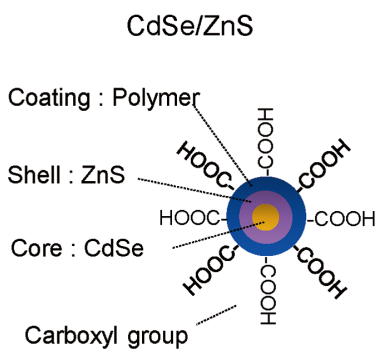

d $\mathrm{Ag}_{2} \mathrm{~S}$

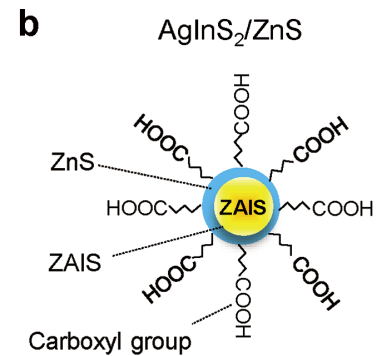

C Graphene e

$\mathrm{AgInTe}_{2}$

\section{$\mathrm{Ag}_{2} \mathrm{~S}$}

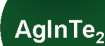

$\operatorname{AginTe}_{2}$

\section{f}

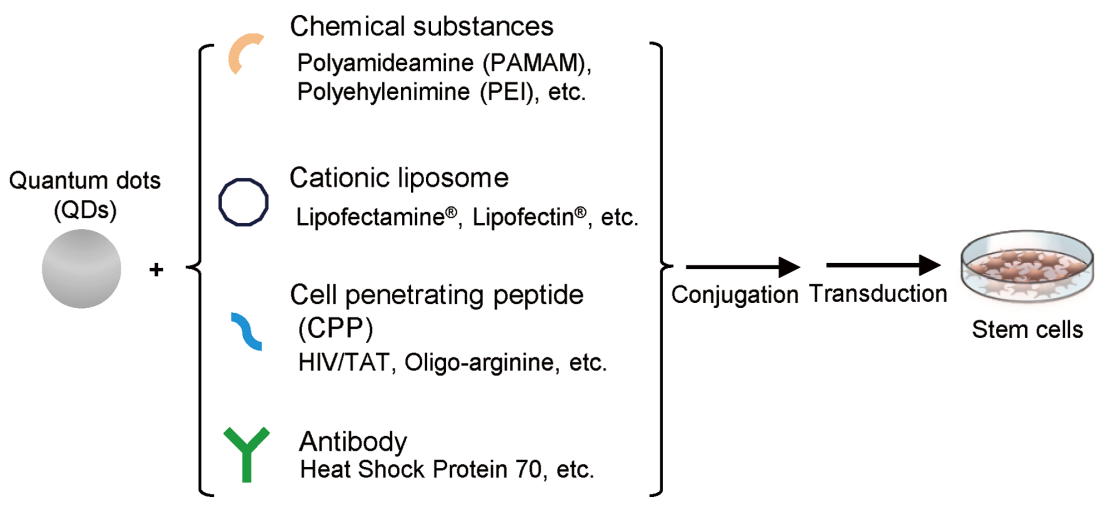

Fig. 1 Illustrations of various kinds of quantum dots (QDs) and QD labeling for stem cells by chemical methods. a: CdSe/ZnS QDs, b: $\mathrm{AgIns}_{2} / \mathrm{ZnS}$ QDs, c: graphene QDs, d: $\mathrm{Ag}_{2} \mathrm{~S}$ QDs, e: $\mathrm{AgInTe}_{2} \mathrm{QDs}$. f: QD labeling for stem cells by chemical methods using chemical substances, cationic liposomes, cell penetrating peptides (CPPs), and antibodies. 
a

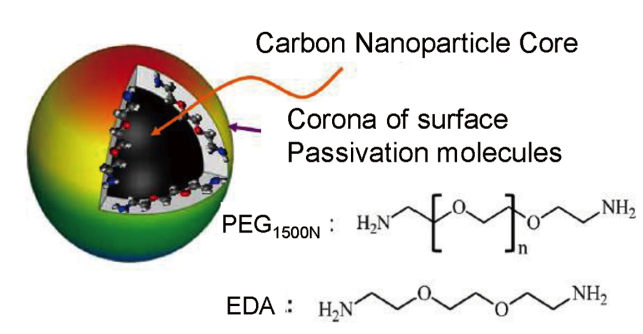

b

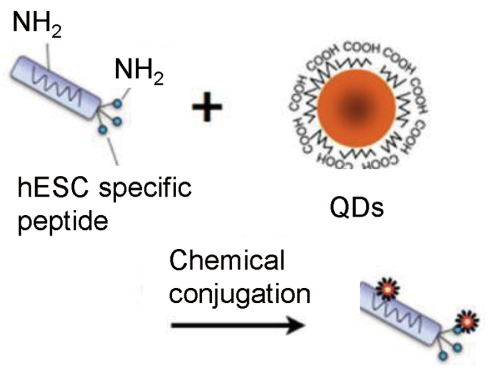

C

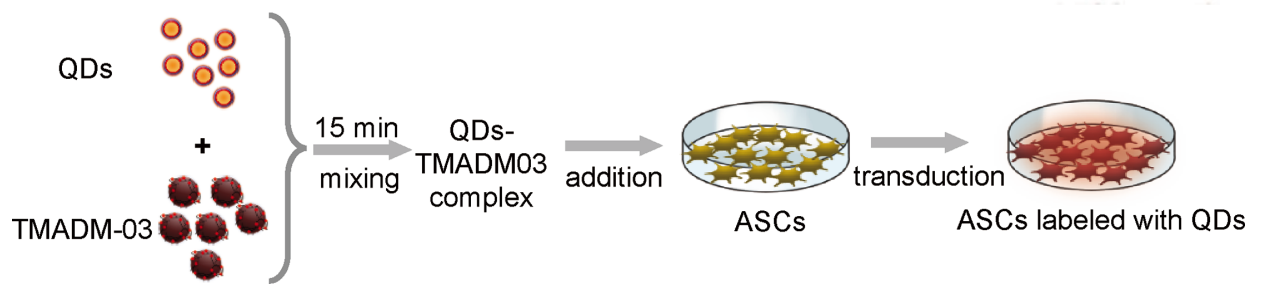

Fig. 2 Surface-modified QDs for cell labeling. a: Carbon illustration on carbon dot, which is generally a small carbon nanoparticle core with attached and strongly adsorbed surface passive molecules. Reprinted with permission from Ref. 35. b: QDs conjugated with phage by chemical bonds between $\mathrm{NH}_{2}$ groups on the phage and the free $\mathrm{COOH}$ group on the QDs. Reprinted with permission from Ref. 42. c: Labeling methods for ASCs by QDs-TMADM-03 complexes. Reprinted with permission from Ref. 43.

quantum yields, superior photostability and wide excitation wavelengths..$^{27-32}$ There are two types of QD structures: coreshell and core-only types. ${ }^{8}$ The core-shell QDs are generally used in bioassays. QDs such as $\mathrm{CdSe} / \mathrm{ZnS}$ and $\mathrm{AgInS}_{2} / \mathrm{ZnS}$ (ZnS-ZAIS) QDs (Figs. 1a and 1b) have been confirmed to be more useful for stem cell labeling than core-only types such as $\mathrm{AgInTe}_{2} \mathrm{QDs}$ (Fig. 1c). ${ }^{33,34}$ In addition, cadmium-free QDs such as graphene QDs and $\mathrm{Ag}_{2} \mathrm{~S}$ QDs (Figs. 1d and 1e) ( $^{35-37}$ have attracted a great deal of attention given the significant decrease in their cytotoxicity against stem cells. For example, the ZnSZAIS QDs developed in our studies were confirmed to have cytotoxicity against stem cells that was about 100 times lower than that of CdSe/ZnS QDs. ${ }^{22}$ Shang et al. onfirmed that GQDs could label human neural stem cells (hNSCs). ${ }^{36}$

Almost all non-toxic organic dyes can easily label stem cells through the cell membrane due to low molecular weight and size by the addition of these dyes to cell culture medium. Labeling technology is very important for the application of QDs to stem cells because of heavy molecular weight and size in comparison to organic probes (Fig. 1f). Chemical methods using chemical substances, cationic liposomes, cell penetrating peptides (CPPs), and antibodies were found to be useful for labeling QDs. ${ }^{15,38-41}$ Octa-arginine (R8) peptide was particularly readily available for stem cell labeling in our studies, because of the low cytotoxicity, easy operation and short transduction time. QDs with a negatively charged surface and R8 peptide were complexed at an optimum concentration ratio for $15 \mathrm{~min}$, and then the solution including QDs and R8 complexes was added to the cell culture medium. After that, QDs were confirmed to be transduced into cells by the endocytosis pathway within at

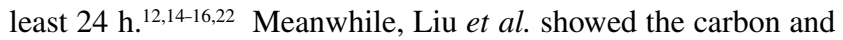
graphene dots with various surface passivation schemes by organic or biomolecules (Fig. 2a). ${ }^{35}$ Sugaya et al. has generated internalizing QDs (i-QDs) by conjugating the particles with an internalizing antibody against mortalin, a heat shock protein 70 family stress chaperone. ${ }^{38}$ Zhao et al. demonstrated that H178 phage can specifically bind to human embryonic stem cells
(hESCs) in vitro using QDs conjugated to the H178 phage (Fig. 2b) ${ }^{42}$ Moreover, our groups revealed that a cationic dextran hydroxypropyltrimethyl chloride-coated magnetic iron oxide nanoparticles (TMADM) was useful as a transduction agent for QDs in stem cell imaging (Fig. 2c). ${ }^{43}$ These labeling methods were very simple and could be widely used for various kinds of stem cells and differentiated mature cells.

\section{Influence of QD Labeling on the Pluripotency of Stem Cells}

Various kinds of stem cells, such as induced pluripotent stem (iPS) cells, ${ }^{44}$ embryonic stem (ES) cells, ${ }^{45}$ bone marrow-derived stem cells (BMSCs) ${ }^{46}$ and adipose tissue-derived stem cells (ASCs), ${ }^{47}$ have been already established. Some of these stem cells are used in clinical pathology in regenerative medicine. In our group, ASCs were confirmed to be labeled with $\mathrm{CdSe} / \mathrm{ZnS}$ QDs655 using R8 peptides with high efficiency (Fig. 3a). The labeled ASCs could be differentiated into adipocytes and osteocytes, and red fluorescence derived from $\mathrm{CdSe} / \mathrm{ZnS}$ QDs655 could be observed in differentiated cells, respectively (Fig. 3b). ${ }^{12,18}$ Sugaya et al. showed that rabbit bone marrow MSCs could be labeled with i-QDs and the labeled MSCs could differentiate into osteoblasts (Fig. 3c). ${ }^{41}$

Shang et al. showed that hNSCs could be labeled with GQDs through endocytosis pathway (Fig. 3d), and GQDs did not affect cell viability, proliferation, metabolism and differentiation (Fig. 3e). ${ }^{36}$ Our research confirmed that ASCs could be labeled with QDs using a positively charged magnetic nanoparticle (TMADM) (Fig. 3f). ${ }^{43}$

In addition, iPS cells could be labeled with CdSe/ZnS QDs655 using R8 peptides with high efficiency and without adverse effects on undifferentiated state and pluripotency. Red and green fluorescence derived from CdSe/ZnS QDs655 and green fluorescence protein (GFP), respectively, were observed from mouse iPS cells (iPS-MEF-Ng-20D-17) labeled with CdSe/ZnS 
a

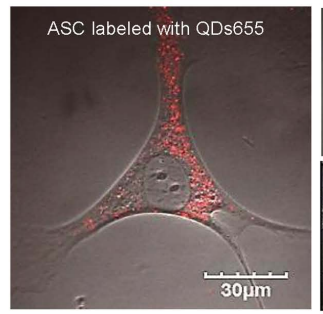

d

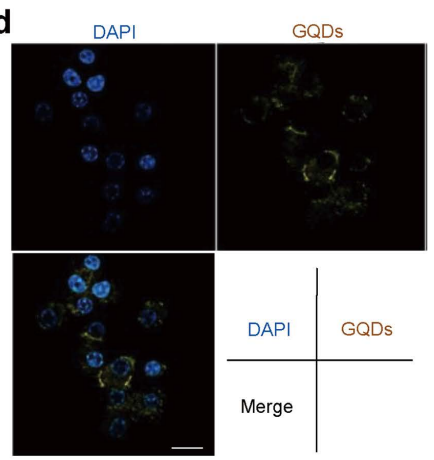

b

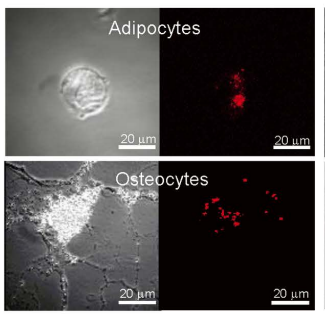

e
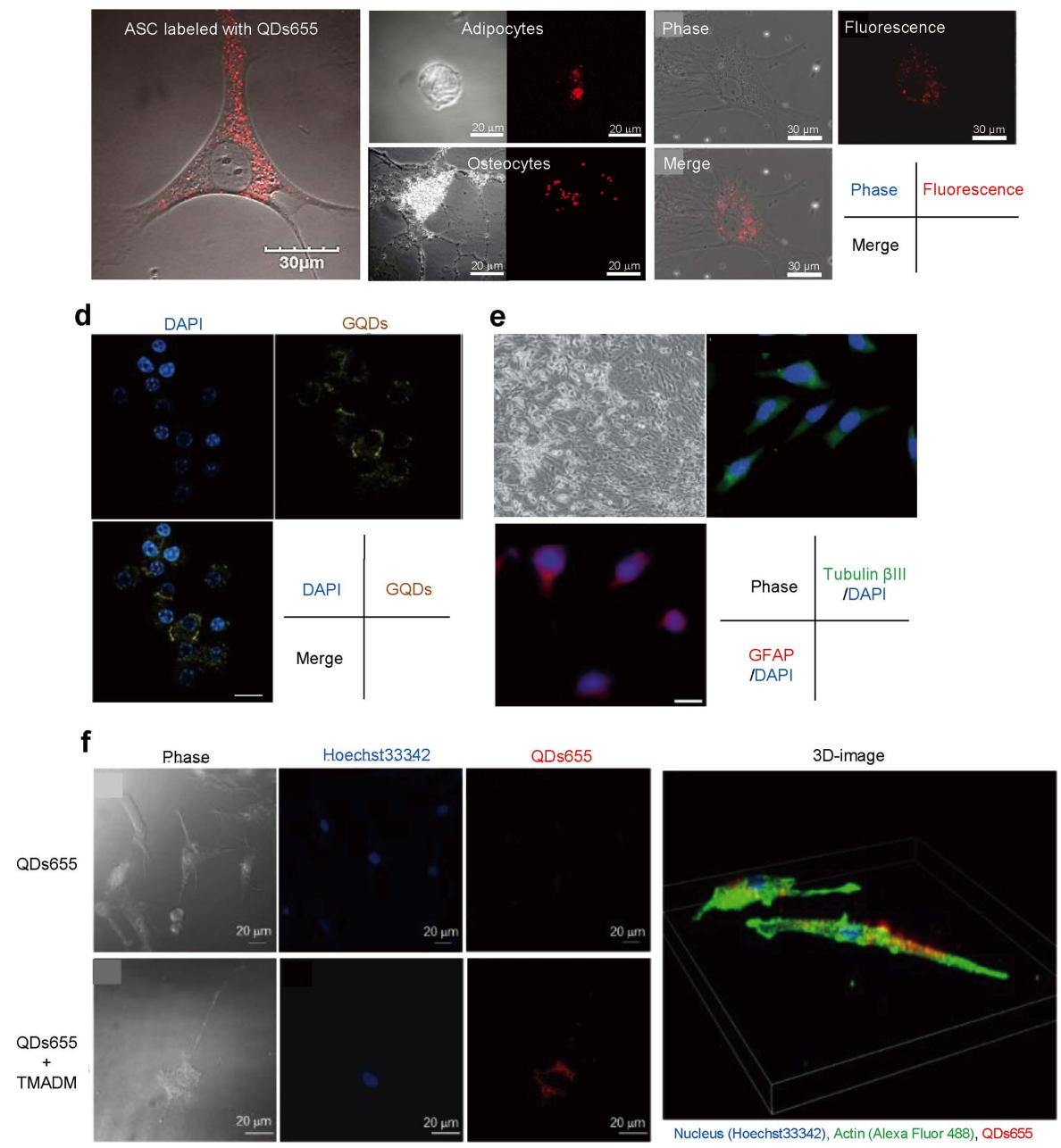

g Phase

QDs655 GFP $\quad \mathbf{h}$
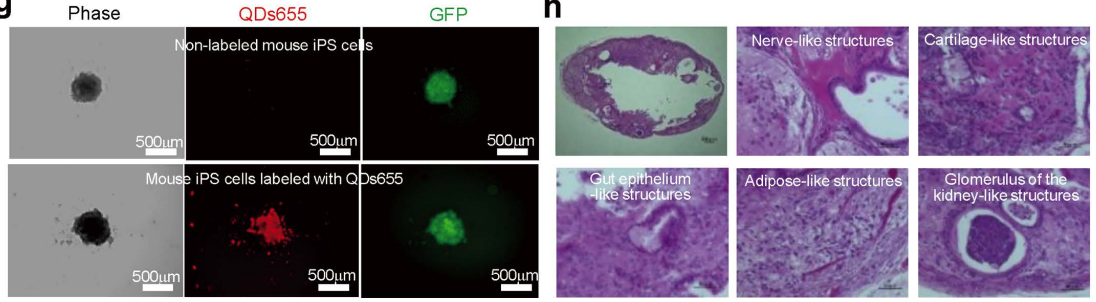

Fig. 3 The fluorescence images of stem cells labeled with QDs and influence of QDs on the pluripotency of stem cells. a: The fluorescence image of ASCs labeled with CdSe/ZnS QDs655 using octa-arginine (R8) peptides. b: The phase and fluorescence images of adipocyte and osteocyte after differentiation of ASCs labeled with CdSe/ZnS QDs655. a, b: Reprinted with permission from Ref. 12. c: The phase and fluorescence images of i-QDs labeled rabbit bone marrow MSCs in vitro. Reprinted with permission from Ref. 41. d: The confocal microscopy images of hNSCs after incubation with GQDs at $37^{\circ} \mathrm{C}$. The scale bar $=20 \mu \mathrm{m}$. e: The bright-field images of the hNSCs differentiated for 14 days. The fluorescence images demonstrated tubulin $\beta \mathrm{III}$ (green) and $\mathrm{GFAP}^{+}$(red) cells. The nuclei were counterstained with DAPI (blue). The scale bar $=20 \mu \mathrm{m}$. d, e: Reprinted with permission from Ref. 41. f: The fluorescence images of ASCs labeled with QDs655 with or without TMADM. Reprinted with permission from Ref. 43. g: The phase and fluorescence images of spheroids of iPS cells labeled with CdSe/ZnS QDs655. h: Teratoma formation of iPS cells labeled with CdSe/ZnS QDs655 after four weeks of injection with labeled iPS cells in nude mice. g, h: Reprinted with permission from Ref. 15.

QDs655 that exhibit green fluorescence derived from GFP in the undifferentiated state (under Nanog gene expression) (Fig. 3g) The teratoma formation of iPS cells labeled with CdSe/ZnS QDs655 has been confirmed after four weeks of injection of labeled iPS cells into nude mice (Fig. 3h). ${ }^{15,18}$

\section{In Vivo Fluorescence Imaging of Stem Cells Labeled with QDs}

A large number of in vivo fluorescence imaging systems have been developed for small and medium-sized animals, and those 
a

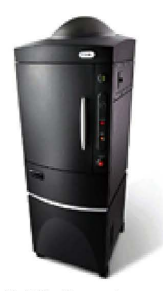

IVIS Spectrum b

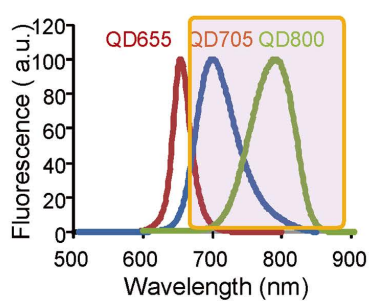

C

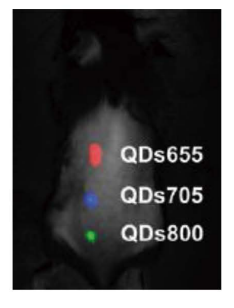

d
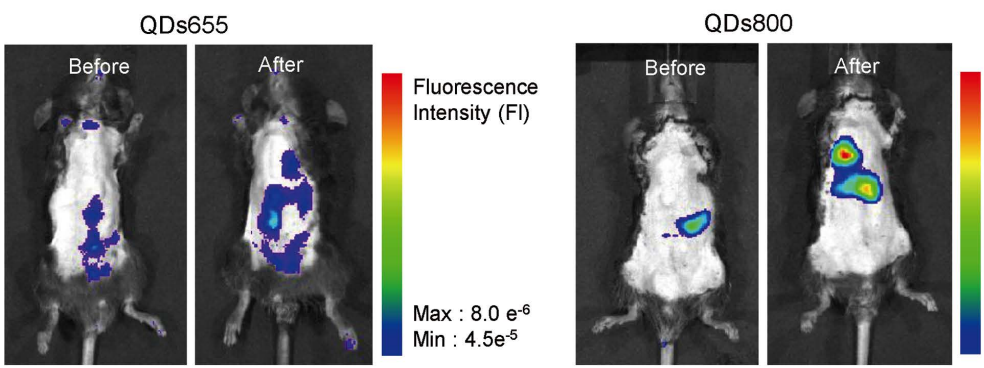

Fluorescence Intensity (FI)
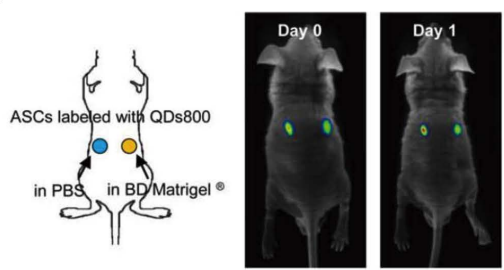

f

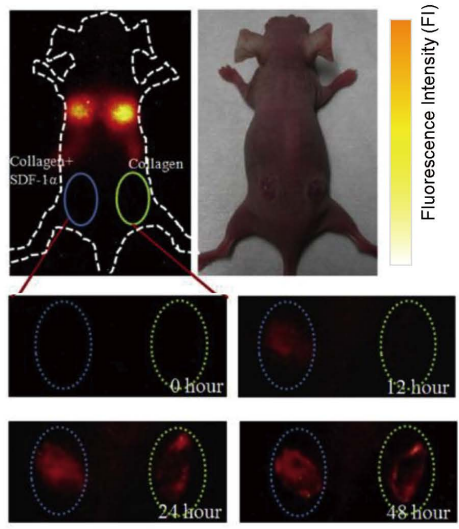

g
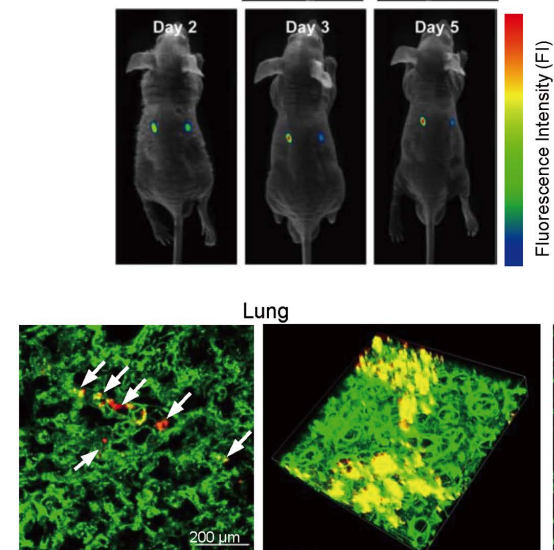

Lung

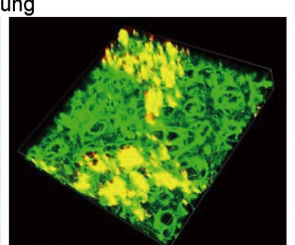

Blood vessel (Isolectin), mASCs (R8-ZZC)

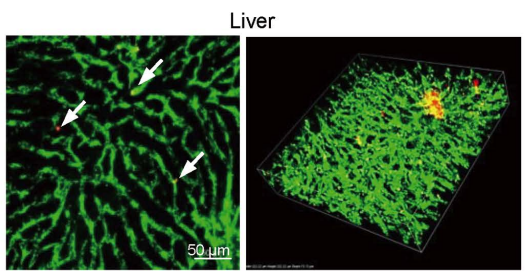

Blood vessel (Isolectin), mASCs (R8-ZZC)

Fig. 4 In vivo fluorescence imaging of transplanted stem cells labeled with QDs. a: In vivo fluorescence imaging system, IVIS Spectrum (PerkinElmer). Reprinted with permission from Ref. 25. b: Fluorescence spectra of CdSe/ZnS QDs655, 705 and 800 in the cell culture medium. c: In vivo fluorescence image of subcutaneously transplanted ASCs labeled with CdSe/ZnS QDs655, 705 and 800 on the backs of mice. b, c: Reprinted with permission from Ref. 12. d: In vivo fluorescence images of mice with acute liver failure after transplantation of ASCs labeled with CdSe/ZnS QDs655 or 800. Reprinted with permission from Ref. 14. e: The pattern diagram of subcutaneous transplantation of ASCs labeled with QDs800 in PBS or BD Matrigel ${ }^{\circledR}$ on the back of a hairless mouse. Reprinted with permission from Ref. 48. f: The photoluminescence images of the migration of hMSCs, which were accumulated at the wounds after $0,4,12,24$, and $48 \mathrm{~h}$ post intravenously injection. Left: wound filled with SDF- $1 \alpha$-loaded collagen, right: wound filled with blank collagen fragments. Reprinted with permission from Ref. 37. g: Multiphoton confocal 2D and 3D images of lung and liver sacrificed from mice after transplantation of ASCs labeled with ZnS-ZAIS QDs. The blood vessels were stained with isolectin conjugated with FITC. Reprinted with permission from Ref. 22.

systems can detect and measure the strength of the fluorescence derived from the body. In our group, IVIS imaging systems such as the IVIS spectrum and IVIS Lumina K series III, have been used for in vivo fluorescence imaging of transplanted stem cells in mice (Fig. 4a). ${ }^{18,25} \mathrm{CdSe} / \mathrm{ZnS}$ QDs705 and 800, which can absorb the excitation light and emit strong fluorescence in the near-infrared (NIR-I; $700-950 \mathrm{~nm}$ ) region, have mainly been used in order to suppress the scattering, absorption and autofluorescence derived from the body (Fig. 4b). However, the advantages of QD fluorescence in the NIR-I region are relatively 
a

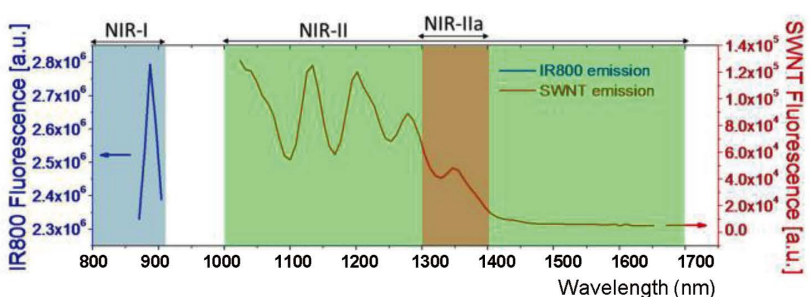

C

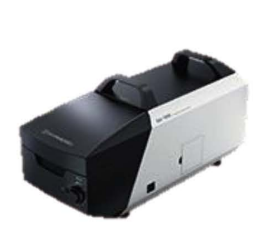

SAl-1000

g

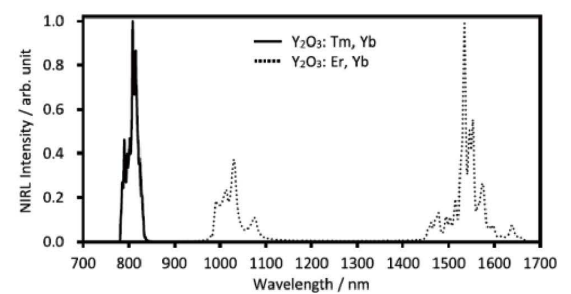

d

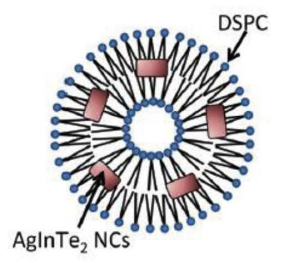

e

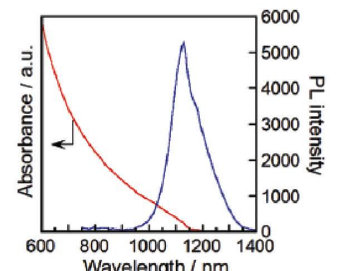

h

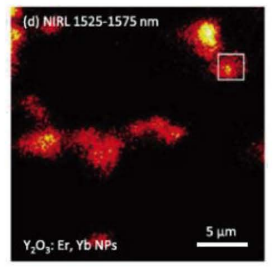

b

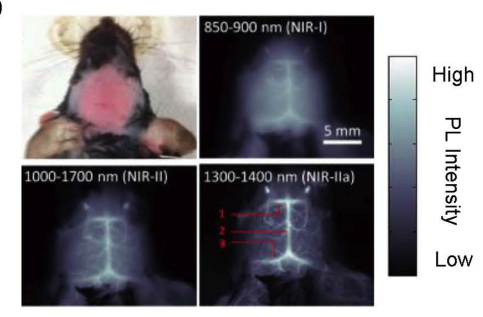

f

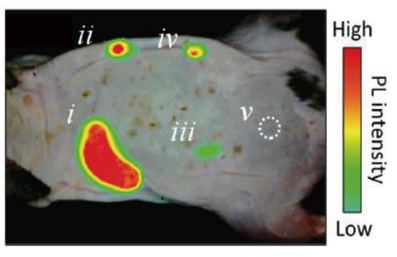

i

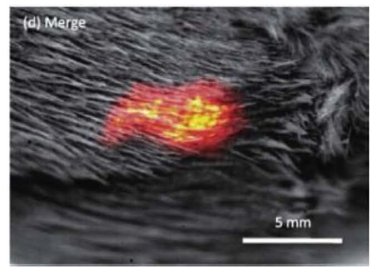

Fig. 5 In vivo NIR-II fluorescence imaging. a: A fluorescence emission spectra of SWNT-IRDye800 conjugate in the range of $850-1650 \mathrm{~nm}$ under the excitation of an 808-nm laser. b: In vivo mouse brain imaging with AWNT-IRDye800 in different NIR sub-regions. a, b: Reprinted with permission from Ref. 49. c: In vivo NIR-II fluorescence imaging system, SAI-1000 (SHIMADZU). Reprinted with permission from Ref. 25. d: Schematic illustration of DSPC-AgInTe ${ }_{2}$ nanocomposite. e: Absorption and fluorescence spectra with DSPC-AgInTe ${ }_{2}$ nanocomposite under $700 \mathrm{~nm}$ excitation. f: In vivo NIRII fluorescence imaging of a mouse injected of DSPC-AgInTe ${ }_{2}$ dispersion. b-d: Reprinted with permission from Ref. 19. g: Normalized fluorescence spectra of $\mathrm{Y}_{2} \mathrm{O}_{3}: \mathrm{Tm}, \mathrm{Yb}$ and $\mathrm{Y}_{2} \mathrm{O}_{3}: \mathrm{Er}, \mathrm{Yb}$ nanoparticles under $980 \mathrm{~nm}$ NIR-II light irradiation. h: NIR-II fluorescence image of $\mathrm{Y}_{2} \mathrm{O}_{3}$ : Er, $\mathrm{Yb}$ nanoparticles under $980 \mathrm{~nm}$ excitation. i: In vivo NIR-II fluorescence imaging of a rat transplanted with a $\mathrm{C} 2 \mathrm{H} 12$ cell sheet labeled with $\mathrm{Y}_{2} \mathrm{O}_{3}$ : Er, $\mathrm{Yb}$ nanoparticles under $980 \mathrm{~nm}$ excitation. e - f: Reprinted with permission from Ref. 20.

few for subcutaneous transplantation, as visible light can pass through the skin to some extent. Indeed, the transplanted ASCs labeled with CdSe/ZnS QDs655, 705 and 800 on the back of mice were able to be detected in three different colors at a high resolution (Fig. 4c). ${ }^{12}$ In contrast, intravenously transplanted ASCs labeled with CdSe/ZnS QDs800 were able to be detected in the lungs and liver without laparotomy; whereas transplanted ASCs labeled with CdSe/ZnS QDs655 were able to not be detected (Fig. 4d). The fluorescence derived from CdSe/ZnS QDs655 in the sacrificed organs from the body could be detected at high contrast, as well as with $\mathrm{CdSe} / \mathrm{ZnS}$ QDs800. ${ }^{14}$

On the other hand, our studies showed that transplanted ASCs labeled with QDs in BD Matrigel $^{\circledR}$ could infiltrate into physiological tissue earlier than in PBS using QDs imaging technology (Fig. 4e) ${ }^{48}$ Chen et al. showed in vivo visualization of the migration of transplanted human mesenchymal stem cells (hMSCs) labeled with $\mathrm{Ag}_{2} \mathrm{~S}$ QDs. The dynamic migration of transplanted hMSCs at the wound site was directly visualized in mice (Fig. 4f). ${ }^{37}$ Furthermore, intravenously transplanted stem cells labeled with QDs that accumulated in organs such as the lungs and liver were able to be detected using a multi-photon excitation confocal laser microscope system such as the A1 $\mathrm{MP}^{+}$ and A1R $\mathrm{MP}^{+}$(Nikon, Tokyo, Japan). ASCs labeled with
ZnS-ZAIS QDs, which had been were intravenously transplanted through the tail vein, were able to be detected in the lungs and liver at the single-cell level. However, the accumulation states of transplanted ASCs were found to differ markedly between the lungs and liver. The transplanted ASCs were observed to aggregate with high efficiency in the lungs, whereas almost all of the ASCs were isolated and few aggregates were observed in the blood vessels of the liver (Fig. 4g). ${ }^{22}$

\section{In Vivo NIR-II Fluorescence Imaging System and Probes}

Fluorescence imaging in the second near-infrared window (NIRII; wavelength $>1000 \mathrm{~nm}$ ) was recently shown to be a good strategy for in vivo imaging of transplanted cells owing to its deeper tissue penetration, lower autofluorescence, and higher spatial and temporal resolution than fluorescence imaging in the NIR-I region. Hong et al. showed the through-skull fluorescence imaging of the brain in an NIR-II using the photoluminescence of single-walled carbon nanotubes (SWNT) (Figs. 5a and 5b). ${ }^{49}$ NIR-II fluorescence, especially NIR-IIa $(1300-1400 \mathrm{~nm})$, could be confirmed to be useful for in vivo 
imaging. However, little progress has been made in the development of in vivo NIR-II fluorescence imaging systems and probes for transplanted cells including stem cells. The SAI1000 developed by SHIMADZU Corporation (SHIMADZU, Kyoto, Japan) is a new in vivo NIR-II fluorescence imaging system that can show high-definition images using the wavelength range of $\geq 1000 \mathrm{~nm}$ (Fig. 5c). ${ }^{25}$ In our group, $\mathrm{AgInTe}_{2} \mathrm{QDs}$ showing a fluorescence peak wavelength of about $1100 \mathrm{~nm}$ were synthesized and transferred into aqueous solution without aggregation using small unilamellar liposomes of 1,2-distearoyl-sn-glycero-3-phosphocholine (DSPC) (Figs. 5d and 5e). DSPC-AgInTe 2 injected into the back of nude mice were able to be detected at a high resolution by a SAI-1000 (Fig. 5f). ${ }^{19}$

In addition, we have focused on lanthanide nanoparticles (LNPs), such as $\mathrm{Y}_{2} \mathrm{O}_{3}: \mathrm{Ln}, \mathrm{Yb}(\mathrm{Ln}=\mathrm{Tm}$ [810 and $1630 \mathrm{~nm}$ ], Er [1530 nm]) nanoparticles with NIR-II fluorescence (Fig. $5 \mathrm{~g}$ ). The observation of NIR-II fluorescence imaging of $\mathrm{Y}_{2} \mathrm{O}_{3}$ : Er, $\mathrm{Yb}$ nanoparticles was successful (Fig. 5h). C2C12 cell sheets labeled with $\mathrm{Y}_{2} \mathrm{O}_{3}$ : Er, $\mathrm{Yb}$ nanoparticles were transplanted onto the backs of the rats, and the NIR-II fluorescence derived from the $\mathrm{C} 2 \mathrm{C} 12$ cells sheets were able to be detected without shearing at a high efficiency (Fig. 5i). ${ }^{20,21}$ However, in vivo NIR-II fluorescence imaging technology, including imaging systems and probes has yet to be established, so further studies are required to apply such a modality to stem cell imaging.

\section{Conclusions}

In this paper, we reviewed our current studies and fields of exploration mainly concerning in vivo fluorescence imaging of transplanted stem cells using QDs. Given that the efficacy of CdSe/ZnS QDs showing NIR-I fluorescence for in vivo imaging of transplanted stem cells has already been confirmed, our imaging technologies for stem cells using QDs have been widely used in many preclinical studies of regenerative medicine. The potential for clinical applications of QDs has expanded with the development of extremely low cytotoxicity QDs lacking heavy metals, such as $\mathrm{Cd}$, as well as the exploitation of NIR-II fluorescence QDs with strong body permeability.

QDs have recently proven to be a novel method for sensing temperature in living cells based on the imaging technique of fluorescence of QDs. Fluorescence imaging-based thermometry was thought to utilize the temperature dependent change of emission intensity, emission lifetime, or emission wavelength at maximum intensity. These techniques have revealed the temperature distribution or event-driven temperature elevation in a cell. However, few of these techniques have been applied for stem cell diagnosis. ${ }^{50}$ In addition, hybrid QDs conjugated or interacted with other functional molecules, such as magnetic nanoparticles, gold nanoparticles and organic molecules, have already been developed. These techniques are expected to enable the simultaneous detection and diagnosis of transplanted stem cell in vivo at the same time.

\section{Acknowledgements}

This paper was mainly supported by the Japan Agency for Medical Research and Development (AMED) through its "Research Center Network for Realization of Regenerative Medicine" and partially supported by the Japan Society for the Promotion of Science (JSPS) KAKENHI Grant Numbers JP16K13646 and JP17H02731. We appreciate the help of
Tomoko Arimoto (Nagoya University) on the submission of this paper.

\section{References}

1. V. S. Urbán, L. Kiss, J. Kovács, E. Gócza, V. Vas, E. Monostori, and F. Uher, Stem Cells, 2008, 26, 244.

2. J. T. Daniels, Nature, 2016, 531, 309.

3. S. Dimmeler, S. Ding, T. A. Rando, and A. Trounson, Nat. Med., 2014, 20, 814.

4. H. Yukawa, H. Noguchi, K. Oishi, S. Takagi, M. Hamaguchi, N. Hamajima, and S. Hayashi, Cell Transplant., 2009, 18, 611.

5. S. Falkner, S. Grade, L. Dimou, K. K. Conzelmann, T. Bonhoeffer, M. Götz, and M. Hübener, Nature, 2016, 539, 248.

6. Y. Xie, T. Yin, W. Wiegraebe, X. C. He, D. Miller, D. Stark, K. Perko, R. Alexander, J. Schwartz, J. C. Grindley, J. Park, J. S. Haug, J. P. Wunderlich, H. Li, S. Zhang, T. Johnson, R. A. Feldman, and L. Li, Nature, 2009, 457, 97.

7. P. Alivisatos, Nat. Biotechnol., 2004, 22, 47.

8. X. Michalet, F. F. Pinaud, L. A. Bentolila, J. M. Tsay, S. Doose, J. J. Li, G. Sundaresan, A. M. Wu, S. S. Gambhir, and S. Weiss, Science, 2005, 307, 538.

9. P. Alivisatos, W. Gu, and C. Larabell, Annu. Rev. Biomed. Eng., 2005, 7, 55.

10. K. Bourzac, Nature, 2013, 493, 283.

11. H. Yukawa, S. Mizufune, C. Mamori, Y. Kagami, K. Oishi, N. Kaji, Y. Okamoto, M. Tokeshi, H. Noguchi, M. Hamaguchi, N. Hamajima, Y. Baba, and S. Hayashi, Cell Transplant., 2009, 18, 591.

12. H. Yukawa, Y. Kagami, M. Watanabe, N. Kaji, Y. Okamoto, M. Tokeshi, N. Noguchi, Y. Miyamoto, Y. Baba, N. Hamajima, and S. Hayashi, Biomaterials, 2010, 31, 4094.

13. Y. Takasaki, M. Watanabe, H. Yukawa, A. Sabarudin, K. Inagaki, N. Kaji, Y. Okamoto, M. Tokeshi, Y. Miyamoto, H. Noguchi, T. Umemura, S. Hayashi, Y. Baba, and $\mathrm{H}$ Haraguchi, Anal. Chem., 2011, 83, 8252.

14. H. Yukawa H, M. Watanabe, N. Kaji, Y. Okamoto, M. Tokeshi, Y. Miyamoto, H. Noguchi, Y. Baba, and S. Hayashi, Biomaterials, 2012, 33, 2177.

15. H. Yukawa, K. Suzuki, Y. Kano, T. Yamada, N. Kaji, T. Ishikawa, and Y. Baba, Cell Med., 2013, 6, 83.

16. Y. Miyazaki, H. Yukawa, H. Nishi, Y. Okamoto, N. Kaji, T. Torimoto, and Y. Baba, Cell Med., 2013, 6, 91.

17. H. Yukawa, M. Watanabe, N. Kaji, and Y. Baba, Cell Med., 2015, 7, 75 .

18. D. Onoshima, H. Yukawa, and Y. Baba, Adv. Drug Deliv. Rev., 2015, 95, 2.

19. T. Kameyama, Y. Ishigami, H. Yukawa, T. Shimada, Y. Baba, T. Ishikawa, S. Kuwabata, and T. Torimoto, Nanoscale, 2016, 8, 5435.

20. S. Fukushima, T. Furukawa, H. Niioka, M. Ichimiya, D. Onoshima, H. Yukawa, Y. Baba, J. Miyake, M. Ashida, and M. Hashimoto, Sci. Rep., 2016, 6, 25950.

21. D. T. K. Dung, S. Fukushima, T. Furukawa, N. Hirohiko, T. Sannomiya, K. Kobayashi, H. Yukawa, Y. Baba, M. Hashimoto, and J. Miyake, Nanomaterials, 2016, 6, 163.

22. Y. Ogihara, H. Yukawa, T. Kameyama, H. Nishi, D. Onoshima, T. Ishikawa, T. Torimoto, and Y. Baba, Sci. Rep., 2017, 7, 40047.

23. S. S. Pillai, H. Yukawa, D. Onoshima, V. Biju, and Y. Baba, Anal. Sci., 2017, 33, 137.

24. Y. Ogihara, H. Yukawa, D. Onoshima, and Y. Baba, Anal. 
Sci., 2017, 33, 143.

25. H. Yukawa and Y. Baba, Anal. Chem., 2017, 89, 2671.

26. R. Doi, T. Tsuchiya, N. Mitsutake, S. Nishimura, M. Matsuu-Matsuyama, Y. Nakazawa, T. Ogi, S. Akita, H. Yukawa, Y. Baba, N. Yamasaki, K. Matsumoto, T. Miyazaki, R. Kamohara, G. Hatachi, H. Sengyoku, H. Watanabe, T. Obata, L. E. Niklason, and T. Nagayasu, Sci. Rep., 2017, 7, 8447.

27. I. L. Medintz and H. Mattoussi, Phys. Chem. Chem. Phys., 2009, 11, 17.

28. W. R. Algar and U. J. Krull, Anal. Bioanal. Chem., 2010, 398, 2439.

29. Z. Deng, Y. Zhang, J. Yue, F. Tang, and Q. Wei, J. Phys. Chem. B, 2007, 111, 12024.

30. W. R. Algar and U. J. Krull, Langmuir, 2008, 25, 633.

31. X. Wu, H. Liu, J. Liu, K. N. Haley, J. A. Treadway, J. P. Larson, N. Ge, F. Peale, and M. P. Bruchez, Nat. Biotechnol., 2003, 21, 41 .

32. W. Liu, M. Howarth, A. B. Greytak, Y. Zheng, D. G. Nocera, A. Y. Ting, and M. G. Bawendi, J. Am. Chem. Soc., 2008, 130, 1274.

33. S. C. Hsieh, F. F. Wang, C. S. Lin, Y. J. Chen, S. C. Hung, and Y. J. Wang, Biomaterials, 2006, 27, 1656.

34. Q. M. Pi, W. J. Zhang, G. D. Zhou, W. Liu, and Y. Cao, BMC Biotechnol., 2010, 10, 36.

35. J. H. Liu, L. Cao, G. E. LeCroy, P. Wang, M. J. Meziani, Y. Dong, Y. Liu, P. G. Luo, and Y. P. Sun, ACS Appl. Mater. Interfaces, 2015, 7, 19439.

36. W. Shang, X. Zhang, M. Zhang, Z. Fan, Y. Sun, M. Han, and L. Fan, Nanoscale, 2014, 6, 5799.

37. G. Chen, F. Tian, C. Li, Y. Zhang, Z. Weng, Y. Zhang, R. Peng, and Q. Wang, Biomaterials, 2015, 53, 265.
38. T. Amna, H. Van Ba, M. Vaseem, M. S. Hassan, M. S. Khil, Y. B. Hahn, H. K. Lee, and I. H. Hwang, Appl. Microbiol. Biotechnol., 2013, 97, 5545.

39. J. Kim, S. H. Song, Y. Jin, H. J. Park, H. Yoon, S. Jeon, and S. W. Cho, Nanoscale, 2016, $8,8512$.

40. J. Qiu, D. Li, X. Mou, J. Li, W. Guo, S. Wang, X. Yu, B. Ma, S. Zhang, W. Tang, Y. Sang, P. R. Gil, and H. Liu, $A d v$. Healthc. Mater, 2016, 5, 702.

41. H. Sugaya, H. Mishima, R. Gao, S. C. Kaul, R. Wadhwa, K. Aoto, M. Li, T. Yoshioka, T. Ogawa, N. Ochiai, and M. Yamazaki, Cytotherapy, 2016, 18, 198.

42. W. Zhao, L. Jin, H. Yuan, Z. Tan, C. Zhou, L. S. Li, and L. Ma, Sci. Rep., 2013, 3, 3134.

43. Y. Ogihara, H. Yukawa, D. Onoshima, and Y. Baba, Anal. Sci., 2017, 33, 143.

44. J. T. Dimos, K. T. Rodolfa, K. K. Niakan, L. M. Weisenthal, H. Mitsumoto, W. Chung, G. F. Croft, G. Saphier, R. Leibel, R. Goland, H. Wichterle, C. E. Henderson, and K. Eggan, Science, 2008, 321, 1218.

45. K. Okita and T. Ichisaka, Nature, 2007, 448, 313.

46. B. S. Shah, P. A. Clark, E. K. Moioli, M. A. Stroscio, and J. J. Mao, Nano Lett., 2007, 7, 3071.

47. M. J. Seo, S. Y. Suh, Y. C. Bae, and J. S. Jung, Biochem. Biophys. Res. Commun., 2005, 328, 258.

48. H. Yukawa, M. Watanabe, N. Kaji, and Y. Baba, Cell Med., 2014, 7, 75 .

49. G. Hong, S. Diao, J. Chang, A. L. Antaris, C. Chen, B. Zhang, S. Zhao, D. N. Atochin, P. L. Huang, K. I. Andreasson, C. J. Kuo, and H. Dai, Nat. Photonics, 2014, $8,723$.

50. R. Tanimoto, T. Hiraiwa, Y. Nakai, Y. Shindo, K. Oka, N. Hiroi, and A. Funahashi, Sci. Rep., 2016, 6, 22071. 